\title{
Estimulación del nervio vago en pacientes migrañosos
}

\author{
Antonio J. Mosqueira, Lydia López-Manzanares, Beatrice Canneti, Alejandro Barroso, \\ Eduardo García-Navarrete, Antonio Valdivia, José Vivancos
}

\begin{abstract}
Introducción. La estimulación del nervio vago (ENV) está aprobada para el tratamiento de la epilepsia refractaria cuando no es posible cirugía resectiva, con una eficacia bien establecida. Series publicadas sugieren un efecto beneficioso de la ENV en la migraña.

Objetivos. Determinar el grado de mejoría de la cefalea en pacientes migrañosos a los que se les había implantado una ENV para tratamiento de la epilepsia refractaria y evaluar qué variables se asocian a mayor posibilidad de éxito con esta medida.
\end{abstract}

Pacientes y métodos. Estudio observacional y retrospectivo desde el 1 de enero de 1999 hasta el 31 de diciembre de 2010. Se contactó telefónicamente con los pacientes con ENV para epilepsia refractaria, seleccionando a aquéllos que cumplían los criterios de la Sociedad Internacional de Cefaleas para la migraña. Se recogieron edad, género, año de implantación, edad de inicio de la epilepsia y la migraña, mejoría de crisis y de migraña, presencia de aura migrañosa y coexistencia de síndrome ansiosodepresivo. Se contactó con 94 pacientes con ENV y se seleccionó a 13 pacientes migrañosos.

Resultados. Tras la implantación de la ENV, se observó una disminución de al menos el 50\% de los episodios de migraña en nueve pacientes (69\%) ( $p=0,004)$, así como una disminución del número de episodios de migraña en aquellos pacientes que también habían reducido sus crisis epilépticas $(p=0,012)$. No se observaron asociaciones estadísticamente significativas en cuanto al sexo, edad, tiempo de evolución, existencia de aura migrañosa o coexistencia de síndrome ansiosodepresivo.

Conclusiones. La ENV podría resultar beneficiosa en pacientes con migraña, especialmente en casos de difícil control. Debido al tipo estudio, hay que tomar estas conclusiones con precaución. Serán necesarios estudios clínicos prospectivos antes de llevarse a la práctica clínica habitual.

Palabras clave. Antiepilépticos. Epilepsia. Estimulación del nervio vago. Migraña.

\section{Introducción}

La prevalencia de la migraña en la población general es de alrededor del $12 \%$, presentando asociación familiar al menos el $60 \%$ de los casos.

La relación entre la migraña y la epilepsia se supone bidireccional. El riesgo de desarrollar migraña está incrementado en pacientes epilépticos [1] y el riesgo de crisis no provocadas aumenta en migrañosos (especialmente, migraña con aura) [2,3].

Clínicamente, tanto la epilepsia como la migraña se caracterizan por síntomas paroxísticos y afectan a individuos que están libres de ataques entre las crisis. En ocasiones pueden existir dificultades para diferenciar una entidad de la otra [4]. La observación directa del episodio y el registro crítico electroencefalográfico permiten resolver la duda y diferenciar ambos procesos, incluso si uno sucede al otro [5].

Se sabe que los síntomas migrañosos implican a múltiples estructuras anatómicas. En el núcleo caudal del trigémino y en el núcleo ventroposterome- dial del tálamo convergen aferencias algésicas desde el cerebro y vasos sanguíneos meníngeos. El hipotálamo regula funciones autonómicas, como el impulso (craving) de comer glúcidos. El sistema límbico desempeña un papel en las alteraciones emocionales como la irritabilidad. La corteza cerebral es el lugar de hiperexcitabilidad notificado en la migraña [6]. Estas estructuras están directa o indirectamente conectadas al núcleo del tracto solitario, que recibe la sensibilidad visceral del nervio vago.

Se considera que un 30-40\% de pacientes epilépticos siguen teniendo crisis pese al tratamiento farmacológico, de los cuales alrededor de un $5 \%$ podría beneficiarse del tratamiento quirúrgico [7]. La comisión de la Liga Internacional contra la Epilepsia define la epilepsia refractaria como aquella en la que se ha producido el fracaso con dos ensayos de fármacos antiepilépticos (FAE), en monoterapia o en combinación, bien elegidos y empleados de forma adecuada para conseguir la ausencia mantenida de crisis [8].
Servicio de Medicina Preventiva; Hospital de Denia (A. Valdivia). Servicio de Neurología (A.J. Mosqueira, L. López-Manzanares, B. Canneti, A. Barroso, J. Vivancos); Servicio de Neurocirugía (E. García-Navarrete); Hospita Universitario de la Princesa; Instituto de Investigación Sanitaria Princesa. Madrid, España.

Correspondencia:

Dra. Beatrice Canneti Heredia. Servicio de Neurología. Hospital Universitario de la Princesa. Instituto de Investigación Sanitaria Princesa. Diego de León, 62. E-28006 Madrid.

E-mail:

canneti.heredia@gmail.com

Aceptado tras revisión externa: 18.04.13.

Cómo citar este artículo: Mosqueira AJ, López-Manzanares L, Canneti B, Barroso A, GarcíaNavarrete E, Valdivia A, et al. Estimulación del nervio vago en pacientes migrañosos. Rev Neurol 2013; 57: 57-63.

(c) 2013 Revista de Neurología 
Tabla I. Relación de estudios publicados respecto a la eficacia de la estimulación del nervio vago (ENV) en las cefaleas.

\begin{tabular}{lccc}
\hline & Indicación de ENV & NN. o de pacientes & Dirección del estudio \\
\hline Basic et al [20] & Epilepsia & 1 & Prospectivo \\
\hline Cecchini et al [22] & Cefalea & 4 & Prospectivo \\
\hline Lenaerts et al [19] & Epilepsia & 10 & Retrospectivo \\
\hline Mauskop [21] & Cefalea & 6 & Prospectivo \\
\hline Hord et al [18] & Epilepsia & 4 & Retrospectivo \\
\hline Sadler et al [17] & Epilepsia & 1 & Retrospectivo
\end{tabular}

La ENV está aprobada para el tratamiento de la epilepsia refractaria con crisis parciales o generalizadas que interfieren en la vida diaria y que haya evolucionado durante un período de tiempo razonable cuando no es posible realizar una cirugía resectiva. Consiste en la estimulación del nervio vago izquierdo a través de electrodos que transmiten una serie de impulsos eléctricos intermitentes, originados en un generador implantado subcutáneamente en la zona infraclavicular.

La eficacia de la ENV en las crisis epilépticas está bien establecida, pero los mecanismos por los cuales la ENV controla las crisis no están dilucidados por completo. Parece que la estimulación provoca un efecto activador o inhibidor descendente desde el núcleo del tracto solitario: sistema reticular activador, tálamo, hipotálamo, complejo amigdalino-hipocampal y corteza cerebral $[9,10]$. El núcleo del tracto solitario proyecta al núcleo caudal del trigémino, que, como ya se ha comentado, está involucrado en la fisiopatología de la migraña.

La ENV crónica e intermitente reduce la frecuencia e intensidad de crisis en humanos, se tolera bien [11] y puede permitir la reducción del número de fármacos o su dosis al aumentar su eficacia con el tiempo de implantación [12]. La cirugía para implantar el dispositivo conlleva escasos riesgos.

En animales, los efectos antinociceptivos de la activación de las aferencias vagales se han demostrado mediante estímulos eléctricos, químicos y fisiológicos [13-16].

La idea de poder tratar la migraña mediante la ENV en humanos se ha estudiado a través de series de pacientes a los que se les había implantado una ENV, donde se observaba que muchos mejoraban clínicamente de sus ataques migrañosos, y se apoya en diferentes hechos fisiopatológicos y epidemiológicos. La mayor parte son series de pacientes con comorbilidad epiléptica y migrañosa a los que se les habían implantado una ENV como tratamiento antiepiléptico y en los cuales, por medio de estudios retrospectivos, se ha visto un efecto beneficioso sobre las migrañas [17-20].

Series como las de Mauskop [21] han estudiado de forma prospectiva el efecto de la ENV en pacientes con migraña refractaria a otras terapias, también con buenos resultados. Otras series se han basado en la selección de pacientes con migraña crónica diaria y depresión para el tratamiento mediante ENV [22] (Tabla I).

La ENV puede resultar beneficiosa en pacientes migrañosos, disminuyendo el número de episodios o haciéndolos menos intensos.

El objetivo principal del estudio es determinar en qué grado los pacientes migrañosos a los que se les había implantado una ENV para tratamiento de la epilepsia refractaria han mejorado de su cefalea tras su instauración. Por otro lado, se pretende evaluar qué variables están asociadas a una mayor posibilidad de éxito con esta medida terapéutica.

\section{Pacientes y métodos}

Se realizó un estudio retrospectivo y observacional, recogiendo datos de pacientes intervenidos en nuestro centro hospitalario, el Hospital Universitario de la Princesa, hospital de referencia situado en el área metropolitana de la ciudad de Madrid, que ha atendido tanto a pacientes de nuestra área sanitaria como a pacientes con epilepsia refractaria derivados de distintas comunidades autónomas.

En la Unidad de Cirugía de la Epilepsia (equipo multidisciplinar formado fundamentalmente por neurólogos, neurocirujanos y neurofisiólogos) se seleccionan los posibles candidatos quirúrgicos y se valora la necesidad de realizar estudios invasivos para determinar si podrían beneficiarse de la cirugía, y así llevar a cabo cirugías resectivas curativas, paliativas o bien otras medidas terapéuticas, como la ENV. Aquellos casos de epilepsia refractaria al tratamiento farmacológico que se excluyen de un tratamiento quirúrgico resectivo son los pacientes que se presentan como candidatos a ENV.

Partiendo del total de pacientes tratados con ENV por epilepsia refractaria entre el 1 de enero de 1999 y el 31 de diciembre de 2010, se inició el contacto telefónico con un total de 94 pacientes, que se logró con 81 pacientes, así como una revisión sistemática de las historias clínicas de estos pacientes. Los mo- 
tivos de no contactar con ellos fueron variados, y en su gran mayoría se debieron a un cambio de domicilio y de teléfono. En 13 pacientes no fue posible el contacto directo tras un máximo de cuatro llamadas telefónicas. Un paciente había fallecido con anterioridad por motivos no neurológicos.

Todos los pacientes fueron informados telefónicamente del estudio y se solicitó su participación en él, que todos aceptaron. Se les informó, asimismo, de que su identidad permanecería oculta y sería remplazada por un código numérico, siguiendo la legislación y normativas vigentes. Todos sus datos han sido tratados de un modo estrictamente confidencial en cumplimiento de la Ley Orgánica 15/1999, de Protección de Datos de Carácter Personal.

Trece pacientes fueron incluidos en el estudio por cumplir los criterios de inclusión, sin presentar criterios de exclusión. Mediante la revisión sistemática de las historias clínicas y una entrevista telefónica se recogió el número medio de episodios de migraña al mes en los tres meses previos a la implantación de la ENV y en los seis meses posteriores a ella. En primer lugar, se comparó la frecuencia de episodios de migraña antes y después de la implantación de la ENV, dentro del período estudiado. Se consideró como pacientes respondedores a los que sufrían una reducción de los episodios de al menos un $50 \%$. Posteriormente, se compararon las variables clínicas y demográficas del grupo de pacientes respondedores con el de no respondedores.

\section{Criterios de inclusión}

Se seleccionaron aquellos pacientes que cumplían los criterios diagnósticos de la Sociedad Internacional de Cefaleas para la migraña [23].

\section{Criterios de exclusión}

- Imposibilidad de una correcta anamnesis debido a discapacidad intelectual.

- Imposibilidad de contacto telefónico directo.

- Cambios en los tratamientos farmacológicos antiepilépticos o en cualquier otro tratamiento que pueda tener efecto en la profilaxis de la migraña, en los tres meses previos y seis meses posteriores a la implantación del estimulador.

- Cefaleas relacionadas únicamente con el período peri o poscrítico.

\section{Hoja de recogida de datos}

Por medio de una entrevista telefónica y de la revisión sistemática de las historias clínicas, se recogie- ron los siguientes datos: área sanitaria de referencia, edad (en el momento del inicio de las migrañas, en el del diagnóstico de epilepsia y en el actual), género, tiempo de seguimiento tras la implantación de la ENV, tipo de crisis epiléptica, comorbilidad psiquiátrica (presencia de síndrome ansiosodepresivo u otros), número de crisis epilépticas (antes y después de la implantación de la ENV), número de episodios migrañosos (tres meses antes y seis meses después de la implantación de la ENV) y presencia de aura migrañosa.

\section{Análisis estadístico}

Todos los análisis se hicieron con el programa Intercooled Stata 9.1 para Windows.

Las variables cualitativas se describieron con la proporción de pacientes en cada categoría. La distribución de las variables cuantitativas se analizó con el test de Shapiro-Wilk, describiéndolas con mediana y rango intercuartílico en caso de desviación significativa de la normalidad, y media y desviación estándar en caso contrario.

La comparación entre número de crisis antes y después de la implantación de la ENV se realizó con el test de Wilcoxon para datos emparejados.

La comparación de variables cualitativas entre respondedores y no respondedores se realizó con tablas de contingencia y el test exacto de Fisher. Para la comparación de variables cuantitativas se utilizó el test de Mann-Whitney. En cada caso se realizó una representación gráfica con diagramas de cajas.

El nivel de significación estadística de las diferencias se fijó en un valor $p<0,05$.

\section{Resultados}

\section{Tamaño muestral}

Durante el período comprendido desde 1999 hasta 2010 se recogieron un total de 94 pacientes a los que se les implantó una ENV para tratar una epilepsia refractaria, de los cuales fue posible contactar con 81 pacientes $(86 \%)$ y fue imposible la comunicación con 13 (13,82\%). De los 81 pacientes, 20 presentaban un grado de discapacidad que imposibilitaba una correcta anamnesis $(24,69 \%)$, por lo que fueron excluidos, quedando, por tanto, 61 pacientes con ENV y posibilidad de una correcta anamnesis de la cefalea. Se seleccionó un total de 13 pacientes migrañosos que cumplían los criterios de inclusión del estudio y que no presentaban criterios de exclusión. 
Tabla II. Características basales de los pacientes seleccionados.

Respondedores

Paciente 1 Paciente 2 Paciente 3 Paciente 4 Paciente 5 Paciente 6 Paciente 7 Paciente 8 Paciente 9 Paciente 10 Paciente 11 Paciente 12 Paciente 13

\begin{tabular}{|c|c|c|c|c|c|c|c|c|c|c|c|c|c|}
\hline Edad & 31 años & 35 años & 29 años & 39 años & 46 años & 34 años & 42 años & 20 años & 38 años & 43 años & 38 años & 29 años & 34 años \\
\hline Sexo & Varón & Varón & Mujer & Varón & Mujer & Mujer & Mujer & Mujer & Mujer & Mujer & Mujer & Mujer & Varón \\
\hline $\begin{array}{l}\text { Edad de inicio } \\
\text { de la epilepsia }\end{array}$ & 4 años & 6 años & 9 años & 17 años & 14 años & 6 años & 6 años & 8 años & 6 años & 13 años & 10 años & 20 años & 13 años \\
\hline $\begin{array}{l}\text { Edad de } \\
\text { implantación } \\
\text { de la ENV }\end{array}$ & 23 años & 25 años & 22 años & 33 años & 43 años & 32 años & 40 años & 18 años & 28 años & 35 años & 31 años & 23 años & 33 años \\
\hline $\begin{array}{l}\text { FAE y dosis } \\
\text { diaria }(\mathrm{mg})\end{array}$ & $\begin{array}{c}\text { CBZ (600) } \\
\text { PB (100) } \\
\text { VPA (1500) }\end{array}$ & $\begin{array}{l}\text { LEV }(500) \\
\text { VPA }(900) \\
\text { PGB }(300) \\
\text { CBZ }(600)\end{array}$ & $\begin{array}{l}\text { PHT (300) } \\
\text { LEV (2000) } \\
\text { TPM (600) } \\
\text { PGB }(300)\end{array}$ & $\begin{array}{c}\text { CBZ }(1.200) \\
\text { VPA }(3.500) \\
\text { CZP }(1,5) \\
\operatorname{TGB}(45)\end{array}$ & $\begin{array}{c}\text { LEV }(1.500) \\
\text { PB (200) } \\
\text { CLB (30) }\end{array}$ & $\begin{array}{c}\text { VPA }(1.500) \\
\text { CZP }(1,5) \\
\operatorname{LTG}(300)\end{array}$ & $\begin{array}{c}\text { LEV (1.000) } \\
\text { PB (50) } \\
\text { CLB (20) }\end{array}$ & $\begin{array}{c}\text { LEV }(1.000) \\
\text { CZP (1) } \\
\text { ZNS (400) } \\
\text { VPA }(1.000)\end{array}$ & $\begin{array}{c}\text { PGB }(600) \\
\text { CBZ }(1.000) \\
\text { CZP }(1,5)\end{array}$ & $\begin{array}{c}\text { VPA (1.000) } \\
\text { CZP (1) } \\
\text { LTC (100) }\end{array}$ & $\begin{array}{c}\text { TPM (600) } \\
\text { PGB (300) } \\
\text { PB (200) }\end{array}$ & $\begin{array}{l}\text { VPA }(1.500) \\
\text { OXC }(1.200)\end{array}$ & $\begin{array}{c}\text { VPA (1.500) } \\
\text { LTG (100) } \\
\text { ZNS (400) } \\
\text { CLB (30) }\end{array}$ \\
\hline $\begin{array}{l}\text { Aura } \\
\text { migrañosa }\end{array}$ & No & Sí & No & No & No & No & No & No & No & No & No & No & No \\
\hline
\end{tabular}

CBZ: carbamacepina; CLB: clobazam; CZP: clonacepam; ENV: estimulación del nervio vago; FAE: fármacos antiepilépticos; LEV: levetiracetam; LTC: lamotrigina; PB: fenobarbital; PGB: pregabalina; PHT: fenitoína; TGB: tiagabina; TPM: topiramato; VPA: ácido valproico.

\section{Características de la muestra (Tabla II)}

Edad: media, $35 \pm 6,9$ años; mediana, 35 años (rango intercuartílico, RIC: 31-39). Sexo de los pacientes evaluados: mujeres, 69\%; hombres, 31\%. Edad de implantación de la ENV: media, 29,7 \pm 7,4 años; mediana, 31 años (RIC: 23-33). Edad de inicio de la migraña: media, 17,8 \pm 4,1 años; mediana: 18 años (RIC: 15-20). Edad de inicio de la epilepsia: media, 10,2 \pm 4,9 años; mediana, 9 años (RIC: 6-13). El tiempo de evolución de la epilepsia presenta una distribución no distinta de una normal (ShapiroWilk: $p=0,169)$ : media, $25 \pm 7,9$ años. El tiempo de evolución de las migrañas también presenta una distribución no distinta de una normal (Shapiro-Wilk: $p=0,878)$ : media, $17,3 \pm 8$ años.

\section{Resultados del análisis}

La distribución del número de migrañas preimplantación de ENV es significativamente distinta de una normal (Shapiro-Wilk: $p=0,013$ ). La mediana de episodios al mes de migraña antes de la implantación fue de 4 (RIC: 2-8). La mediana de episodios al mes de las migrañas tras la implantación de la ENV fue de 1 (RIC: 1-2).

$\mathrm{Al}$ ser muestras emparejadas, han sido comparadas con el test de Wilcoxon: tras la implantación de la ENV se observa una disminución de al menos el $50 \%$ de los episodios de migraña en nueve pacientes (69\%). Las diferencias observadas fueron estadísticamente significativas $(p=0,004)$ (Fig. 1).

Si la representación se estratifica según si el paciente tuvo mejoría en las crisis epilépticas (1) o no (0), los diagramas de cajas quedarían según se señala en la figura 2.

En el grupo de pacientes que mejoraron de las crisis epilépticas, la disminución del número de migrañas fue estadísticamente significativa (test de Wilcoxon: $p=0,012$ ).

Se observa una tendencia en este sentido también entre los pacientes en quienes no existió mejoría en las crisis epilépticas, aunque no se alcanza significación estadística $(p=0,162)$ (en este subgru- 
po sólo se incluyeron cuatro pacientes, lo que obliga a tomar los datos con precaución).

Al categorizar la respuesta de las migrañas, se observa una asociación próxima a la significación estadística entre mejoría de crisis epilépticas y mejoría de crisis de migraña (test exacto de Fisher: $p=$ 0,052). Es 24 veces más probable mejorar de las migrañas si se ha mejorado de la epilepsia que si no se ha mejorado (intervalo de confianza al 95\% de la odds ratio $=1,11-518,58)$.

No hay diferencia significativa de edad entre los que mejoran de migrañas y los que no $(p=0,642)$. No existe asociación significativa entre sexo y mejoría de migrañas tras implantación de la ENV (test exacto de Fisher: $p=0,176)$. Como dato curioso (aunque no significativo, dado el reducido tamaño muestral), todos los varones $(n=4)$ mejoraron de su migraña tras la implantación de la ENV, y sólo mejoraron cinco de las mujeres. El tiempo de evolución de la migraña no se asocia significativamente con la mejoría (o falta de mejoría) de las migrañas tras implantar la ENV (test de Mann-Whitney: $p=$ 0,877). El tiempo de evolución de la epilepsia no se asocia significativamente con la mejoría (o falta de mejoría) de migrañas tras implantar la ENV (test de Mann-Whitney: $p=0,816$ ). La existencia de síndrome ansiosodepresivo no se asocia significativamente con la mejoría (o falta de mejoría) de la migraña (test exacto de Fisher: $p=1$ ).

\section{Discusión}

Nuestros resultados parecen indicar que la ENV puede resultar beneficiosa en pacientes migrañosos, principalmente en pacientes con epilepsia refractaria cuya epilepsia responde a la ENV.

Las teorías actuales sugieren que el inicio de los ataques de migraña implica una disfunción primaria del sistema nervioso central, con la subsiguiente activación del sistema trigémino vascular [24]. La inhibición de las neuronas nociceptivas en el núcleo del trigémino por la ENV podría desempeñar un papel en los mecanismos antimigrañosos. Estudios retrospectivos en pacientes epilépticos tratados con ENV sugieren que puede tener un papel en el tratamiento de la migraña.

En el estudio retrospectivo de Hord et al [18] se observa una disminución en la intensidad en el número y en la frecuencia de los episodios migrañosos. Esta mejoría se observa de forma precoz en los tres primeros meses tras la implantación del dispositivo, como sucede en nuestra serie. También de forma congruente con nuestros resultados, la serie
Figura 1. Frecuencia de migrañas antes y después de implantar la estimulación del nervio vago (ENV).

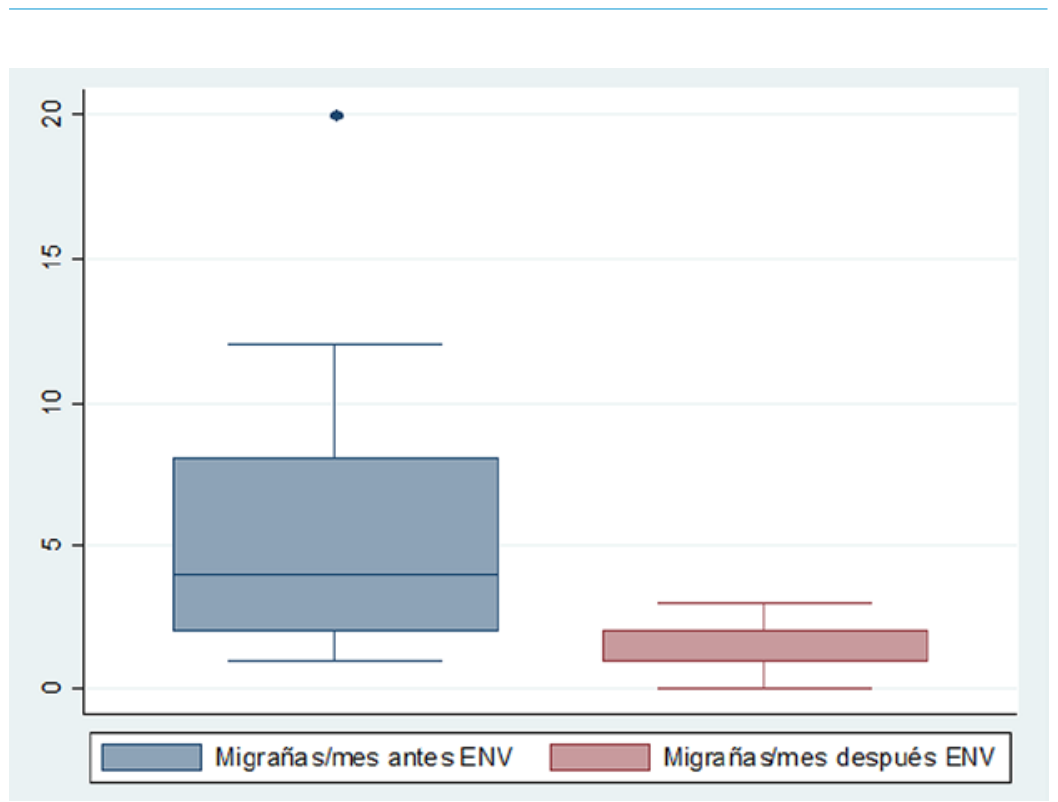

Figura 2. Frecuencia de migrañas antes y después de implantar la estimulación del nervio vago (ENV) comparando el grupo sin mejoría de la epilepsia (izquierda) y el grupo con mejoría de la epilepsia (derecha).

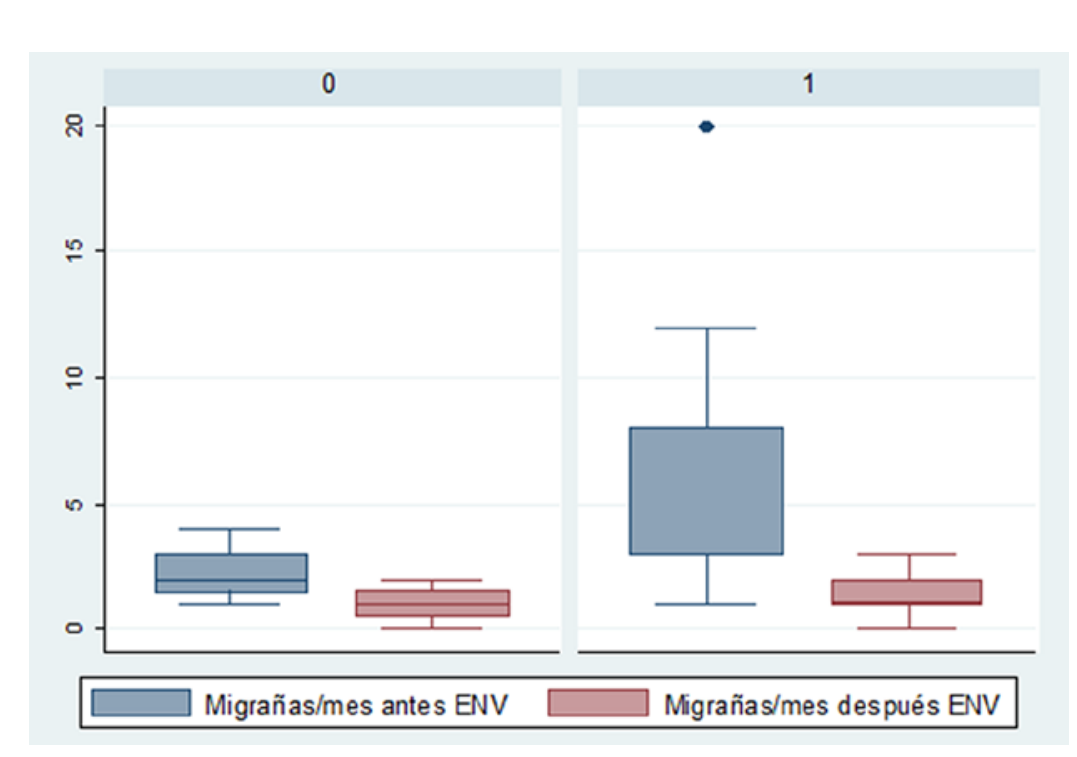

de Sadler et al [17] muestra una mejoría en la frecuencia de episodios de cefalea en los dos primeros meses. Series como la de Mauskop [21] y casos aislados publicados refuerzan la hipótesis de que la 
ENV puede ser eficaz en el tratamiento de la migraña crónica (Tabla I).

Sin embargo, las características de diseño de este estudio hacen que las conclusiones extraídas deban ser interpretadas con precaución.

En primer lugar, los FAE tienen utilidad como tratamiento preventivo de la migraña. Actualmente existe evidencia de la utilidad del ácido valproico, topiramato, gabapentina, lamotrigina y zonisamida para la profilaxis de la migraña [25]. Muchos de los pacientes epilépticos que padecen migraña están tratados con alguno de estos fármacos, motivo por el cual pueden estar recibiendo profilaxis contra la migraña de forma indirecta. Se considera que se debe esperar un mínimo de 12 semanas para determinar la eficacia de estos fármacos como profilaxis de migraña [26].

El hecho de que haya muchos pacientes migrañosos que están recibiendo tratamiento con algún FAE debido a una comorbilidad con la epilepsia hace que esto pueda suponer un sesgo de confusión, que impida sacar conclusiones válidas acerca del efecto de la ENV sobre la migraña. Para controlar este sesgo se ha excluido a pacientes a los que, durante el período de tiempo del estudio, se les introdujo alguno de estos tratamientos en los meses anteriores y posteriores a la implantación del estimulador, de modo que se pueda aislar su efecto sobre las migrañas. Los pacientes incluidos en el estudio no sufrieron modificaciones en los FAE ni en sus dosis durante el período de éste.

El tamaño muestral es de sólo 13 pacientes, lo que hace que debido a la falta de potencia estadística, alguna de las asociaciones buscadas no sea estadísticamente significativa.

El hecho de que sea un estudio realizado en pacientes tratados con ENV por epilepsia refractaria, después de haber sido seleccionados por presentar una epilepsia refractaria, sin tener en cuenta la presencia o no de migrañas en estos criterios de selección, se debe a que no existe indicación específica para la implantación de ENV en pacientes migrañosos, lo que puede, a su vez, sesgar los resultados.

La única variable de las analizadas que presenta una asociación estadísticamente significativa con la mejoría de las migrañas tras la implantación de la ENV es la existencia de una mejoría en cuanto al número de crisis epilépticas. Esto se puede interpretar como que la ENV actúa sobre factores fisiopatológicos desconocidos y comunes a un subgrupo de epilepsias y de migrañas, que haga que sean más susceptibles de mejora. Por otro lado, estos resultados podrían deberse a que se trate de pacientes 'satisfechos' con la intervención, que tiendan a auto- evaluar su presente estado de salud de una forma más positiva que aquellos en los cuales la ENV no ha sido efectiva.

Debido, probablemente, al reducido número de pacientes, no ha sido posible establecer una asociación estadísticamente significativa entre la ENV y el resto de variables analizadas (sexo, tiempo de evolución de la migraña y de la epilepsia a la hora de implantar la ENV, coexistencia de un síndrome ansiosodepresivo), aunque alguna de ellas muestra tendencias interesantes, como que mejoraron el 100\% de varones frente al $55 \%$ de mujeres.

La ENV parece influir de forma positiva en pacientes con comorbilidad epilepsia-migraña, al reducir de una forma estadísticamente significativa los episodios de migraña. Serán necesarios más estudios, especialmente prospectivos y aleatorizados, para evaluar la eficacia de este tratamiento y la posibilidad de emplearlo, especialmente en pacientes con migraña rebelde a otras terapias.

\section{Bibliografía}

1. Ottman R, Lipton RB. Comorbidity of migraine and epilepsy. Neurology 1994; 44: 2105-10.

2. Hauser WA, Annegers JF, Kurland LT. Prevalence of epilepsy in Rochester, Minnesota; 1940-1980. Epilepsia 1991; 32: 429-45.

3. Ludvigsson P, Hesdorffer D, Olafsson E, Kjartansson O,

Hauser WA. Migraine with aura is a risk factor for unprovoked seizures in children. Ann Neurol 2006; 59: 210-3.

4. Alberca R. Epilepsia y jaqueca. Rev Neurol 1998; 26: 251-5.

5. Marks DA, Ehrenberg BL. Migraine-related seizures in adults with epilepsy, with EEG correlation. Neurology 1993; 43: 2476-83.

6. Ambrosini A, Maertens de Noordhout A, Sandor PS, Schoenen J. Electrophysiological studies in migraine: a comprehensive review and their interest and limitations. Cephalalgia 2003; 23 (Suppl 1): S13-31.

7. Rosenow F, Lüders H. Presurgical evaluation of epilepsy. Brain 2001; 124: 1683-700.

8. Kwan P, Arzimanoglou A, Berg AT, Brodie MJ, Allen Hauser W, Mathern G, et al. Definition of drug resistant epilepsy: consensus proposal by the ad hoc Task Force of the ILAE Commission on Therapeutic Strategies. Epilepsia 2010; 51: 1922.

9. Schachter SC, Saper CB. Vagus nerve stimulation. Epilepsia 1998; 39: 677-86.

10. Morris GL, Mueller WM. Long-term treatment with vagus nerve stimulation in patients with refractory epilepsy. Neurology 1999; 53: 1731-5.

11. Ramsay RE, Uthman BM, Augustinsson LE, Upton ARM, Naritoku D, Willis J, et al; First International Vagus Nerve Stimulation Study Group. Vagus nerve stimulation for treatment of partial seizures: safety side effects, and tolerability. Epilepsia 1994; 35: 627-36.

12. Ardesch JJ, Buschman HP, Wagener-Schimmel LJ, Van der Aa HE, Hageman G. Vagus nerve stimulation for medically refractory epilepsy: a long-term follow-up study. Seizure 2007; 16: 579-85.

13. Tfelt-Hansen P, Pascual J, Ramadan N, Dahlöf C, D’Amico D, Diener HC, et al; International Headache Society Clinical Trials Subcommittee. Guidelines for controlled trials of drugs in migraine: third edition. A guide for investigators. Cephalalgia 2012; 32: 6-38.

14. Chandler MJ, Hobbs SF, Bolser DC, Foreman RD. Effects of vagal afferent stimulation on cervical spinothalamic tract neurons in monkeys. Pain 1991; 44: 81-7. 
15. Randich A, Ren K, Gebhart GF. Electrical stimulation of cervical vagal afferents. II. Central relays for behavioral antinociception and arterial blood pressure decreases. J Neurophysiol 1990; 64: 1115-24.

16. Ren K, Randich A, Gebhart GF. Effects of electrical stimulation of vagal afferents on spinothalamic tract cells in the rat. Pain 1900; 44: 311-9.

17. Sadler RM, Purdy RA, Rahey S. Vagal nerve stimulation aborts migraine in patient with intractable epilepsy. Cephalalgia 2002; 22: 482-4.

18. Hord ED, Evans MS, Mueed S, Adamolekun B, Naritoku DK. The effect of vagus nerve stimulation on migraines. J Pain 2003; 4: 530-4.

19. Lenaerts ME, Oommen KJ, Couch JR, Skaggs V. Can vagus nerve stimulation help migraine? Cephalalgia 2008; 28: 392-5.

20. Basic S, Sporis D, Chudy D, Grahovac G, Nevajda B.

The effect of nerve vagus stimulation on migraine in patient with intractable epilepsy: case report. Neurol Sci 2012; Jun 22. [Epub ahead of print].

21. Mauskop A. Vagus nerve stimulation relieves chronic refractory migraine and cluster headaches. Cephalalgia 2005; 25: 82-6.
22. Cecchini AP, Mea E, Tullo V, Curone M, Franzini A, Broggi G, et al. Vagus nerve stimulation in drug-resistant daily chronic migraine with depression: preliminary data. Neurol Sci 2009; 30 (Suppl 1): S101-4.

23. Headache Classification Committee of the International Headache Society. The international classification of headache disorders. 2 ed. Cephalalgia 2004; 24: 1-160.

24. Edvinsson L. Aspects on the pathophysiology of migraine and cluster headache. Pharmacol Toxicol 2001; 89: 65-73.

25. Aicher SA, Lewis SJ, Randich A. Antinociception produced by electrical stimulation of vagal afferents: independence of cervical and subdiaphragmatic branches. Brain Res 1991; 542: 63-70.

26. Silberstein SD, Holland S, Freitag F, Dodick DW, Argoff C, Ashman E. Evidence-based guideline update: pharmacologic treatment for episodic migraine prevention in adults: report of the Quality Standards Subcommittee of the American Academy of Neurology and the American Headache Society. Neurology 2012; 78: 1337-45.

\section{Vagus nerve stimulation in patients with migraine}

Introduction. Vagus nerve stimulation (VNS) has been approved for the treatment of refractory epilepsy when resective surgery is not possible, and has proved to be highly effective. Series published in the literature suggest a beneficial effect of VNS in the treatment of migraine.

Aims. To determine the degree to which headaches improve in patients with migraine after the placement of VNS to treat refractory epilepsy, and to evaluate what variables are associated with an increased chance of success with this measure.

Patients and methods. An observation-based retrospective study was conducted from 1st January 1999 until 31st December 2010. Patients with VNS for refractory epilepsy were contacted by telephone, after selecting those who fulfilled International Headache Society criteria for migraine. Data collected included age, gender, year of placement, age at onset of epilepsy and migraine, improvement of seizures and migraine, presence of migraine with aura and coexistence of anxious-depressive syndrome. Ninety-four patients with VNS were contacted and 13 patients with migraine were selected.

Results. Following placement of the VNS, the number of episodes of migraine was seen to decrease by at least $50 \%$ in nine patients $(69 \%)(p=0.004)$ and there was a drop in the number of episodes of migraine in those patients who had also reduced their epileptic seizures $(p=0.012)$. No statistically significant associations were observed as regards sex, age, length of disease history, existence of migraine with aura or coexistence of anxious-depressive syndrome.

Conclusions. VNS could have beneficial effects for patients with migraine, especially in cases that are difficult to control. Due to the type of study, these conclusions must be taken with caution. Prospective clinical studies are needed before introducing the technique into daily clinical practice.

Key words. Antiepileptic drugs. Epilepsy. Migraine. Vagus nerve stimulation. 